\title{
Osteophytes may be a rare cause of recurrent pleural effusions
}

\author{
K. Psathakis, V. Skouras, F. Chatzivassiloglou, K. Tsintiris
}

ABSTRACT: Osteophytes may be a rare cause of recurrent pleural effusions. K. Psathakis, V. Skouras, F. Chatzivassiloglou, K. Tsintiris.

We report a rare case of a 59 year-old female, who was admitted to the hospital because of a recurrent right-sided pleural effusion. The initial work-up was non-diagnostic. The diagnosis was finally confirmed following medical thoracoscopy, where an osteophyte of a rib was found to pro- trude sharply into the thoracic cavity. Chronic inflammatory changes of the pleura suggested mechanical irritation due to long lasting friction between this bony structure and the underlying lung. This is the first report where an osteophyte seems to be implicated in pleural pathology. A brief review of the available data from the literature is presented to further support our results.

Monaldi Arch Chest Dis 2013; 79: 2, 87-89.

Keywords: Osteophytes, Pleural effusions, Medical thoracoscopy.

Department of Pneumonology, Army General Hospital of Athens, Greece.

Correspondence: Kostas Psathakis,MD, Karaoli M. 18,Dafni,17235 Athens, Greece; e-mail: kpsazakis@gmail.com

Little is known about whether or how skeletal deformities of the thoracic cage may be implicated in pleural pathology. To our knowledge this is the first reported case where an osteophyte is considered to be responsible for the formation and the recurrences of a pleural effusion.

\section{Case Report}

A 59 year-old female (ex-smoker, 25 packyear) was admitted to the Pneumonology Department of our Hospital, on $1^{\text {st }}$ October 2012, due to a recurrent right-sided pleural effusion. Her past medical history was unremarkable.

Her present illness started about 10 months prior to admission when she first complained of a dull pain and sense of "heaviness" on her right hemithorax. The chest x-ray (CXR) revealed a small right-sided pleural effusion. Two months earlier she had fallen down the stairs in her house, which resulted in a fracture of the 9th rib on the right-hand side of her rib cage; A CXR at that time had no abnormal findings apart from the fracture. The treating physician suggested non-steroid antiinflammatory medication and follow-up, attributing to the effusion to the prior trauma. Indeed, the symptoms gradually subsided and at follow-up, one month later, the pleural effusion had disappeared from the CXR.

However, three months later, the symptoms reappeared along with a small right-sided pleural effusion. Again, observation and follow-up was suggested since the effusion was considered too small for thoracocentesis. During the following months, symptoms persisted, and pleural fluid amount gradually increased, occupying approximately one-sixth to one-fifth of the right hemithorax.
At that point, the patient was admitted to our hospital for further investigation of the pleural effusion. Physical examination was normal except from the signs of the effusion on the right hemithorax. Routine blood test results as well as serum antinuclear antibodies, rheumatoid factor and complement levels were normal. Diagnostic thoracocentesis was performed and $60 \mathrm{ml}$ of serosanguinous pleural fluid were aspirated. The fluid was a lymphocytic $(60 \%)$ exudate with negative cytology and low levels of adenosine deaminase (ADA) $(16 \mathrm{U} / \mathrm{L})$. Apart from the pleural effusion, no other abnormalities were found on computed tomography $(\mathrm{CT})$ of the chest and abdomen. CT pulmonary angiography was negative for pulmonary embolism.

Due to lack of diagnosis after the initial workup, the patient was subjected to medical thoracoscopy. Exploration of the pleural cavity revealed mildly thickened parietal pleura, mainly at the base of the right-hemithorax (lower costal and diaphragmatic pleura), compatible with chronic inflammation. The anterior chest wall surface at the level of the right clavicle was remarkable for a whitish space-occupying lesion with a bony appearance projecting into the pleural cavity. The visceral pleura where the projecting lesion seemed to make contact, was whitish and thickened probably due to the chronic friction and irritation (figure 1). Biopsy specimens from the affected parietal pleura showed no specific findings but chronic inflammation.

Review of the patient's CT-scan detected the aforementioned bony structure which had not previously been reported as it had been considered clinically irrelevant. It was the chondro-sternal joint of the first right rib, which had undergone degeneration and calcification forming an osteo- 
phyte. The osteophyte had an inward orientation toward the thoracic cavity, protruding sharply in the direction of the underlying lung (figure 2).

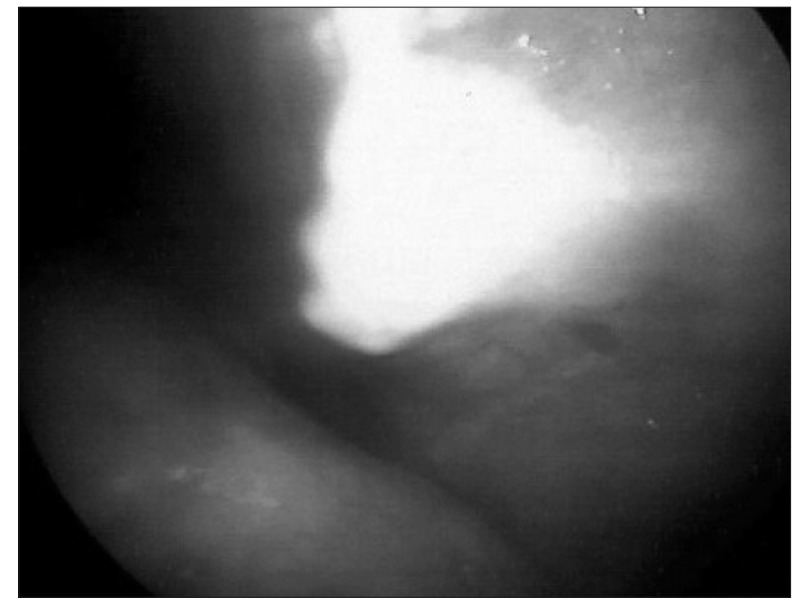

Fig. 1. - Thoracoscopic image: the bony projection is visible as well as the locally thickened visceral pleura (whitish area) of the adjacent lung.

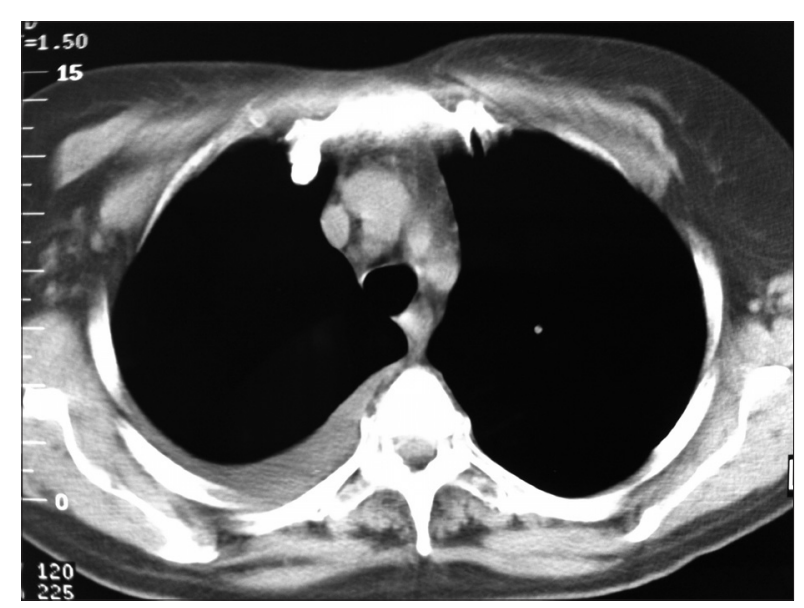

Fig. 2. - A Computed Tomography scan of the chest reveals the inward protruding osteophyte. A small right-sided pleural effusion is also visible.

The patient was discharged on $4^{\text {th }}$ October 2012 with the diagnosis of a pleural effusion of traumatic aetiology. No specific treatment was applied. She is currently on regular follow-up at the out-patient clinic, every three to six months for the following 2 years, without recurrence of the pleural effusion until now.

\section{Discussion}

The most important and unexpected finding in this case was the protruding osteophyte into the pleural cavity. To find a possible explanation of the recurrent pleural effusions in our patient, we performed a literature search on PubMed to find any cases of skeletal deformities, particularly rib-related occurrences, which could be responsible for the development of pleural effusions. No relevant cases were detected using the terms "pleural effusion" combined with one of the following: "osteophytes", "bone spicules", "rib deformities" or "rib anomalies". However, a few cases of rib exostoses (or osteochondromas) causing pleural effusions were found.

It has been proposed that when exostoses are oriented inward toward the thoracic cavity, they may cause mechanical injury to adjacent anatomic structures such as pleura, diaphragm, heart and lungs. The complications that have been reported with such lesions are rare and mainly include hemothorax, pneumothorax, fibrothorax, diaphragm laceration and hemopericardium, depending on their location [1-3]. Hemothorax is the most frequently reported complication of pleural injury caused by these lesions. Two etiologic mechanisms have been implicated: a direct trauma by the sharp tip of exostoses and/or a spontaneous rupture of abnormally dilated pleural blood vessels that resulted from long-standing friction between the intrathoracic exostoses and the visceral pleura [2-4]. Although exostoses (osteochondromas) are a different entity from osteophytes, it would be reasonable to assume that their mechanical effect on adjacent structures is most probably similar since both of them act like space-occupying lesions inside the chest cavity.

Considering exostoses as a paradigm, there is some evidence that suggests that chronic friction of the bony spurs with the adjacent visceral pleura might cause pleural inflammation with or without a pleural effusion [3, 4]. Tomos at el described a case of fibrothorax associated to hereditary multiple exostoses of the ribs, consistent with a chronic, clinically unrecognised, inflammation [1]. In another article, a young male with rib exostoses developed a pleural effusion after "the repeated throwing of a ball" [5]. This case implies that any mechanical stress of the chest (like exercise or trauma) may have an additive effect on pre-existing chronic pleural inflammation caused by the presence of rib anomalies, finally resulting in the formation of pleural effusion. These observations are in accordance with the presented case, where a pleural effusion was developed after an ipsilateral rib fracture.

Moreover, thoracoscopic findings in our patient revealed chronic inflammation of the parietal and thickening of the visceral pleura that came into contact with the protruding ostephyte. These data support the hypothesis that in our case, a recurrent pleural effusion developed after a chest trauma, which intensified a pre-existing chronic inflammation of the pleura due to the presence of the osteophyte. Strenuous exercise, such as cycling or gardening (hobbies that our patient practiced regularly), might have been responsible for the recurrence and persistence of the pleural effusion after the initial trauma.

Localized inward-orientated chest wall abnormalities are hardly ever detected upon physical examination by palpation and their diagnosis is most commonly based on imaging. A CT-scan is more sensitive than CXR for the detection of such lesions; moreover, it is an accurate method to establish the nature as well as the exact location of the lesion and its topographic relations to adjacent structures [2]. 
In the case of persistent or recurrent pleural effusion, however, it is mandatory to exclude more common causes of pleural effusion such as malignancy, infection (including tuberculosis) and pulmonary embolism. Following a non-diagnostic initial work-up, thoracoscopy should be considered as the next diagnostic step. In our case, thoracoscopy not only assisted the exclusion of malignancy and tuberculosis but also suggested the diagnosis by allowing for direct visualization of the pleural surface and detection of the protruding lesion.

Regarding costal osteochondromas, in the absence of symptoms, there is no clear indication for surgical treatment. Although most authors have suggested that inward-protruding bony spicules of costal osteochondromas require surgical excision, others have managed them conservatively. In our case we preferred the latter option since the patient remains asymptomatic and, in contrast to osteochondromas, osteophytes do not reveal malignant transformation [2]. Avoidance of strenuous exercise, patient reassurance regarding the benign nature of this condition and observation with regular follow-up might be a sufficient approach.

\section{References}

1. Tomos P, Lachanas E, Pavlopoulos D, Michail OP, Kafetzis DA. An unexpected cause of bilateral hemothorax. Respiration 2010; 79: 152.

2. Assefa D, Murphy RC, Bergman K, Atlas AB. Three faces of costal exostosis. Case series and review of literature. Pediatr Emer Care 2011; 27: 1188-1191.

3. Teijeira FJ, Baril C, Younge D. Spontaneous pneumothorax in a patient with hereditary multiple exostoses. Ann Thorac Surg 1989; 48: 717-718.

4. Reynolds JR, Morgan E. Haemothorax caused by a solitary costal exostosis. Thorax 1990; 45: 68-69.

5. Graham J, Winterson R, Grovell J, Boon RL. An unusual cause of a pleural effusion. Emerg Med J 2008; 25: 749 .

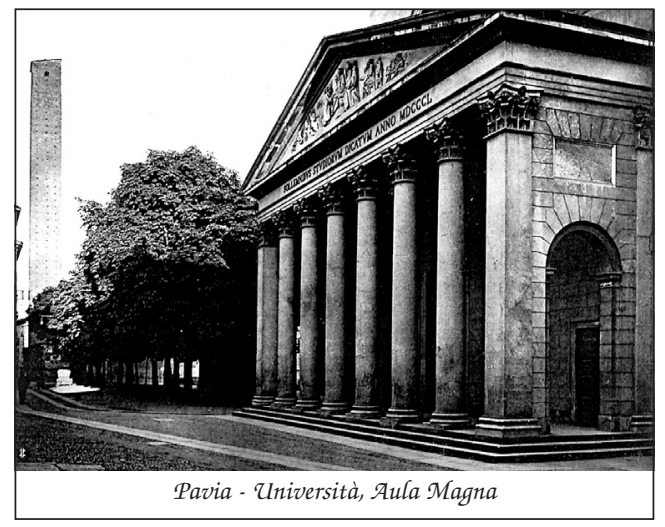

